Int. J. Speleol. 9 (1977/78), pp. 251-266.

\title{
Les Araignées du genre Rhode de Yougoslavie (Araneae, Dysderidae)
}

par

C. L. DEELEMAN-REINHOLD*

\section{SUMMARY}

\section{The spiders of the genus Rhode in Yugoslavia (Araneae, Dysderidae).}

Rhode magnifica $\mathrm{n}$. sp. is described from a Montenegrine cave and Rhode stalitoides $\mathrm{n}$. $\mathrm{sp}$. from a Bosnian cave. There is a redescription of Rhode aspinifera (Nikolić). The author includes in the genus Rhode the species previously contained in the genera Harpassa and Typhlorhode. The northern Y ugoslav genus Stalita and related genera are regarded to be the nearest relatives of $R$ hode and it is concluded that they have originated on the Balkan Peninsula from a common ancestor.

\section{Historique}

Dans son "Histoire naturelle des Araignées", Simon créa le groupe Rhodeae, qui, avec les Dysderae et les Holissae compose la sous-famille des Dysderinae. Les Rhodeae comprenaient deux genres: Rhode et Harpassa. Le genre Rhode fut créé par cet auteur en 1882 pour le Rhode scutiventris épigé des Monts Cantabres en même temps que le genre Harpassa pour H. tenuipes, dont un seul exemplaire mâle avait été trouvé sous des pierres dans les montagnes de Corse. En 1893, Simon décrivit le Rhode biscutata d'Algérie et signala la présence de $R$. scutiventris dans ce même pays.

A cause de la rareté des Rhode dans les collections, probablement causé par une vie cryptique de ces araignées, il s'écoula un très long temps sans parler de Rhode. En 1935, Kratochvil décrivit le premier représentant de Yougoslavie d'après une seule femelle d'une espèce aveugle trouvée par Absolon dans un gouffre du centre de l'Herzégovine, et le plaça dans un troisième genre: Typhlorhode subterranea. A part son excellent travail et une illustration magnifique en couleur, il ne nous reste plus rien de cette découverte: le type fut détruit au cours des bombardements de la ville de Brno pendant la seconde

\footnotetext{
* Sparrenlaan 8, Ossendrecht, Pays-Bas.
} 
guerre mondiale. L'espèce ne fut jamais retrouvée. Cependant, le grand spéléologue Egon Pretner trouva de petits Rhode aveugles dans les environs de Postojna dans les grottes, mais aussi sous des pierres profondement enfoncées dans le fond des forêts. Ils fûrent décrits par Nikolić (1963) sous le nom de Typhlorhode aspinifera, les mâles restent inconnus.

Nos prospections dans les grottes yougoslaves pendant 17 années ne nous ont pas conduit à la redécouverte de $T$. subterranea. Elles ont toutefois abouti à la récolte de deux espèces nouvelles de Rhodeae. Bien que ce matériel ne consiste qu'en un spécimen adulte de chaque espèce, il me semble justifié de les décrire parce qu'elles se distinguent nettement par plusieurs caractères morphologiques.

\section{Systématique}

L'auteur des genres Rhode et Harpassa, Simon (1893a), donne comme caractères distinctifs principaux la présence (Rhode) ou l'absence (Harpassa) de scuta abdominaux et des pattes ambulatoires médiocrement longues (Rhode) ou longues et fines (Harpassa). D'autres caractères différentiels seraient constitués par la disposition des yeux, l'armure des chélicères et la forme des lames-maxillaires, mais ceux-ci se sont révélés être des caractères assez plastiques au sein du groupe. En effet, une étude comparative des types de Harpassa tenuipes avec ceux de $R$. scutiventris et $R$. biscutata me permet de confirmer que les seules différences essentielles entre ces genres sont situées dans la longueur des pattes et dans la configuration de l'abdomen.

Or, l'attribution des deux espèces nouvelles décrites dans le présent travail à l'un ou l'autre genre offre des difficultés unsurmontables. Chacune des espèces nouvelles devrait être rangée dans le genre Rhode (ou Typhlorhode) selon certains caractères, dans le genre Harpassa selon d'autres, les caractères distinctifs étant continus. Leur placement serait tout à fait arbitraire.

Le genre Typhlorhode, créé par Kratochvil (1935) doit son statut de genre à: 1) l'absence de yeux, 2) la présence d'un scutum dorsal chez la femelle et 3) les filières inférieures qui sont au moins aussi longues que les supérieures. 1) Il faut noter que chez les Araignées, on n'accorde plus une valeur de niveau générique au seul caractère d'anophtalmie. 2) Si l'on se rend compte que chez les espèces connues de Rhode la présence et les dimensions des scuta abdominaux varient fortement selon l'espèce et selon le sexe (biscutata, $\hat{\hat{~}}$ dorsal et ventral entier, $q$ dorsal absent, ventral réduit; scutiventris, $\hat{\delta}$ dorsal absent, ventral presqu'entier, $q$ dorsal absent, ventral réduit), il n'est pas sensé d'établir un nouveau genre sur ce caractère, un genre qui ne s'appuie que sur la morphologie de la femelle seulement. 3) J'ai étudié les filières chez toutes les espèces connues de Rhode sauf subterranea et je n'ai pu constater aucune différence spécifique: chez tous les exemplaires les filières supérieures étant un peu plus longues et grèles que les inférieures.

Pour les raisons exposées ici, je propose de supprimer pour l'instant toute coupure d'ordre générique dans les Rhodeae. Le genre Rhode, qui regroupera 


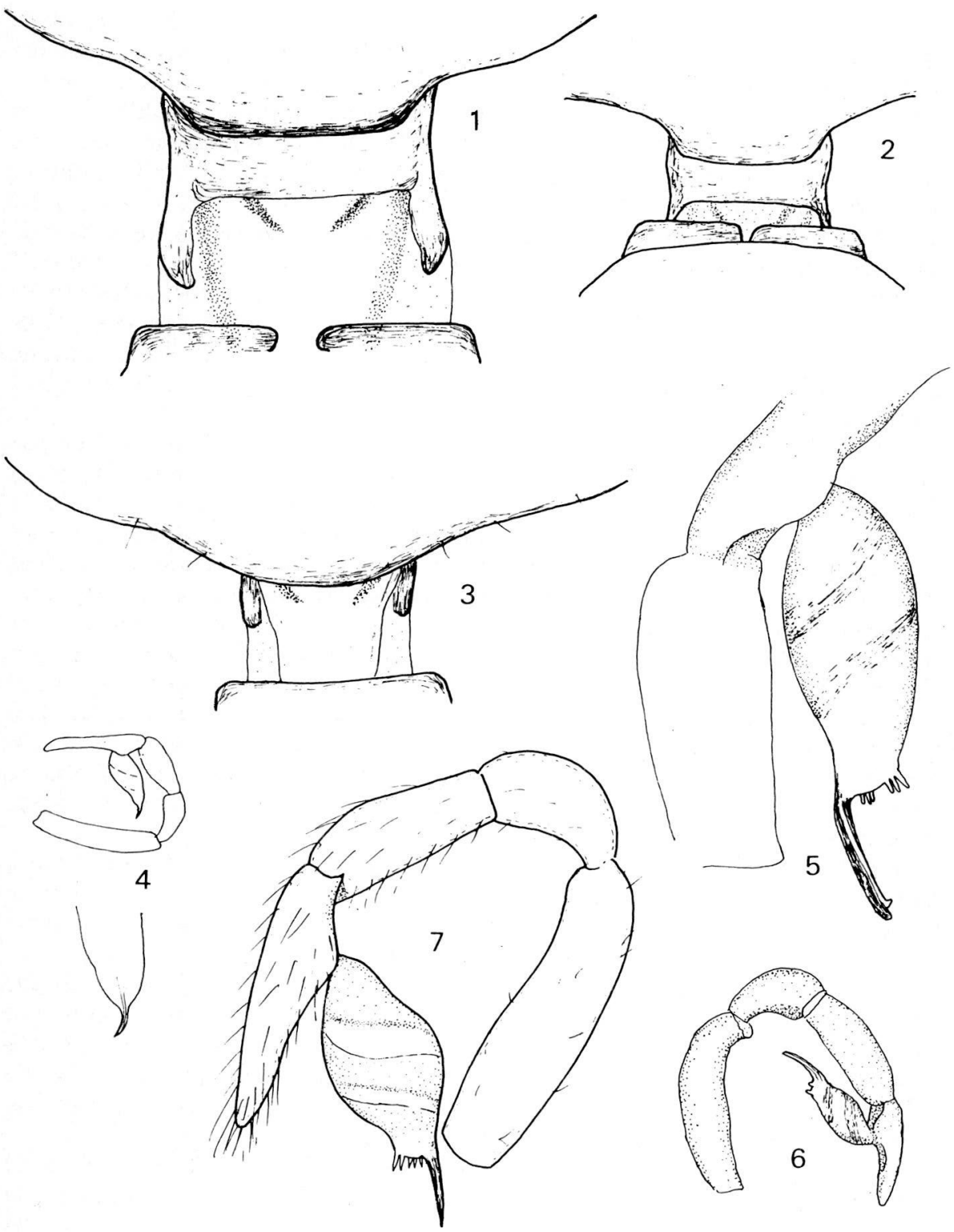

Planche 1. 1) Rhode magnifica n. sp., lobe postérieur du céphalothorax + pédicule -2 ) $R$. tenuipes (Simon), type, id. - 3) R. stalitoides n. sp., id. - 4) R. tenuipes, patte-mâchoire du mâle - 5) $R$. stalitoides, id. - 6) id. - 7) R. scutiventris Simon, Monts cantabres, id. 1-3, 5, 7, x 72,5; 6, x 22 . 
donc les genres Rhode Simon, Harpassa Simon et Typhlorhode Kratochvil, se distingue nettement de tous les autres genres et présente un nombre de caractères morphologiques très distincts. D'ailleurs, dans ce groupe, les récoltes sont si rares et accidentelles qu'on ne peut s'attendre à des découvertes de nouvelles espèces et c'est pourquoi il faut éviter d'en fournir prématurément une diagnose trop rigide. Dans toutes les espèces connues à ce jour, le céphalothorax est assez plat, les stries thoraciques sont presqu'effacées, le bord postérieur est prolongé en lobe au dessus du pédicule. Le sternum est large et convexe, bombé et se prolonge en arrière, souvent enveloppant entièrement la base du pédicule et figurant nettement un pédoncule ralliant le prosoma à l'abdomen. Des bandes chitineuses relient le sternum au céphalothorax entre les coxae des pattes ambulatoires et entre les coxae I et celles des pattes-mâchoires. En outre, une bande chitineuse transversale se trouve entre les pattes-mâchoires et les bases des chélicères. Les pattes sont dépourvues d'épines. Les extrémités des pattes sont munies de tiges qui donnent l'impression d'être constituées par une lanière fasciée qui s'est enroulée hélicoïdalement (planche 6). De telles tiges se trouvent éparpillées sur toutes les parties du corps. Elles semblent beaucoup moins nombreuses chez les Stalita et je n'en ai point observé dans les genres Dysdera, Harpactea et Stalagtia, mais je les ai fréquemment trouvées chez les Mesostalita et chez les Harpactocrates. Elles sont semblables aux „screw-like hairs”, observés et photographiés au microscope à balayage par Lehtinen (1975) dans les extrémités tarsales de Hermippus sp. (Zodariidae) et Tibellus maritimus (Thomisidae). L'abdomen est souvent pourvu d'un scutum dorsal. Ceci leur donne une certaine ressemblance avec les Oonopidae. Les différences les plus saillantes avec ceux-ci résident dans la forme du céphalothorax et des pièces buccales, le sternum, le scutum divisé chez les Oonopidae et les organes copulateurs. Les organes copulateurs de Rhode sont assez uniformes et d'un type représenté sur les planches 1-3.

Parmi les Dysderidae, le genre le plus proche est sans doute Stalita (planche 7) qui a en commun avec Rhode la forme des chélicères, de la pièce labiale, du bord antérieur du sternum et les organes copulateurs; seul le tarse de la patte-mâchoire du mâle est plus long chez Rhode que chez Stalita.

"Harpassa circe" Brignoli, 1975, ne semble posséder, ni le lobe postérieur thoracique, ni la prolongation sternale autour du pédicule. Ceci en conjonction avec les organes copulateurs fort dissemblables à ceux de Rhode, exclut une appartenance au Rhodeae de cette forme. Son bulbe rappelle celui de Harpactea rucnerorum Thaler \& Polenec de Dalmatie, mais la conformation des chélicères et de la vulva l'éloignent de ce dernier.

La présence de Harpassa tenuipes aux environs de Dubrovnik (Nikolić, 1966) doit être contestée. J'ai étudié les exemplaires de "Harpassa" figurant dans la collection de cet auteur provenant de Lapad, Dubrovnik $(1+, 1$ subad. $q)$ Ils se sont avérés être des Harpactea rucnerorum. 


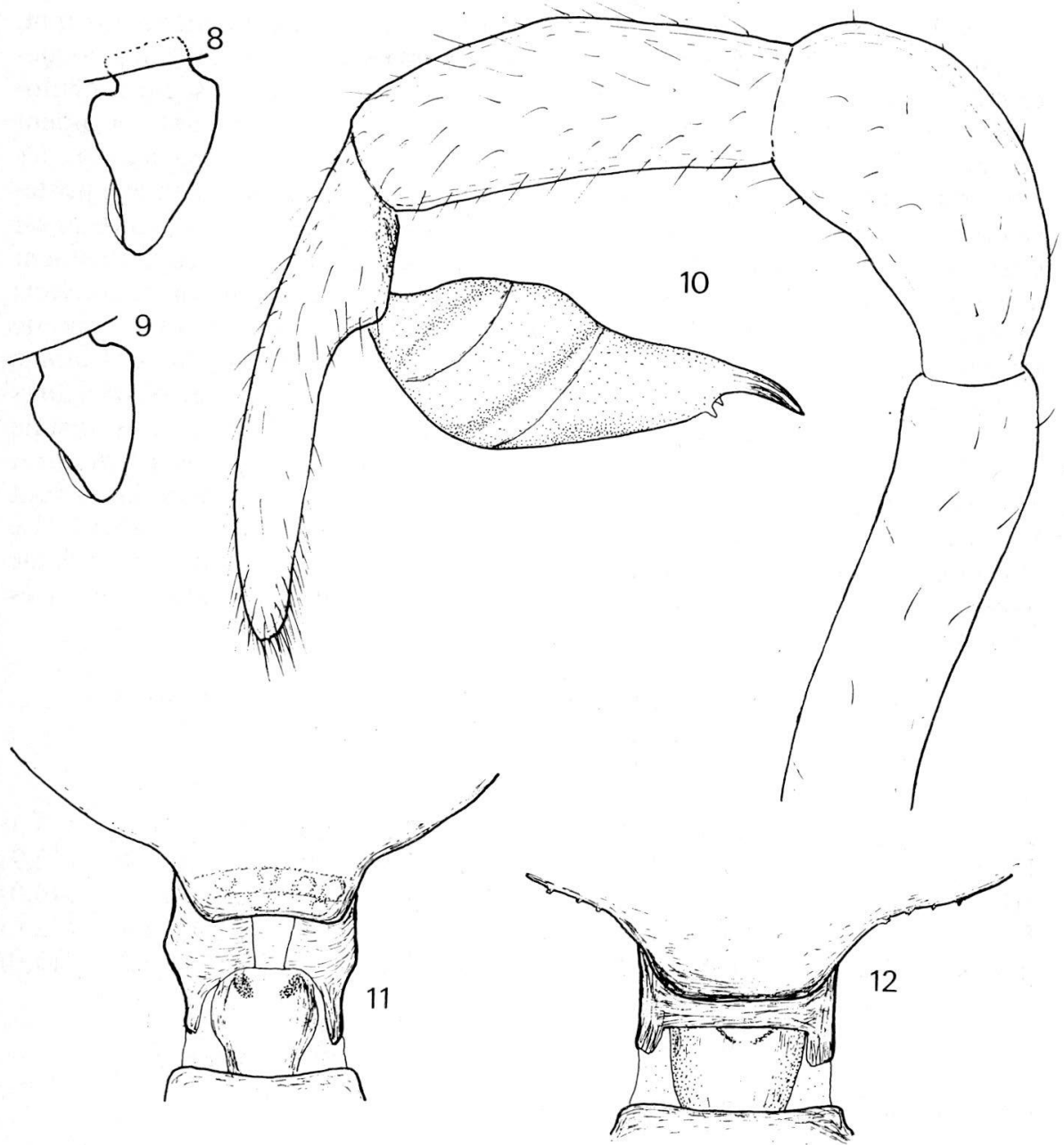

Planche 2. 8) Rhode biscutata Simon, Edough, chélicère droite, face extérieure - 9) R. stalitoides, id. - 10) $R$. biscutata, patte-mâchoire du mâle - 11) $R$. scutiventris $Q$., Monts Catabres, lobe postérieur du céphalothorax + pédicule -12$) R$. biscutata 0 , Edough, id. 8, 9, x 22; 10-12, x 72,5

RHODE MAGNIFICA spec. nova (pl. 1, 1; pl. 3, 15-17; pl. 6, 26)

Grotte glacière "Ledena pećina na Kiti", Trubjela, district de Nikšić, Monténégro. 1 ㅇ , leg. A. P. B. Deeleman, 7-VIII-1969.

Description. - Longueur du céphalothorax + abdomen $6,7 \mathrm{~mm}$, céphalo- 
thorax 2,7 mm long, 2,0 $\mathrm{mm}$ large, largeur de la partie céphalique $1,0 \mathrm{~mm}$, longueur du pédicule $0,5 \mathrm{~mm}$. Le céphalothorax et le sternum sont rougebrunâtres foncés, les autres parties chitinisées sont plus claires. Le céphalothorax est fortement atténué en avant, les yeux presque connivants occupent un peu plus que la moitié de la largeur de la partie céphalique, les antérieurs sont séparés entr'eux d'un tiers de leur diamètre, les médians postérieurs sont un peu plus serrés l'un de l'autre que des latéraux. Le bandeau est égal au diamètre des antérieurs. La strie thoracique est très faiblement développée. Le céphalothorax et la face antérieure des chélicères sont couverts de granulations piligères. Les chélicères ont $1,0 \mathrm{~mm}$ de longueur et $0,5 \mathrm{~mm}$ de largeur, elles sont plus allongées que celles des espèces occidentales et moins fortement géniculées postérieurement. Les marges antérieures et postérieures sont munies de deux dents de dimensions égales, la dent la plus distale séparée des autres par une distance inférieure de moitié à celle de $R$. biscutata et $R$. scutiventris. L'échancrure est diagonale, concave. Les lames-maxillaires sont très légèrement divergentes et ne s'inclinent point sur la pièce labiale. Le sternum est prolongé en arrière en anneau entourant entièrement le pédicule sans suture dorsale, sa surface est grenue réticulée. Les pattes sont très longues et grêles, les mensurations en mm sont les suivantes:

\begin{tabular}{|c|c|c|c|c|c|c|c|c|c|}
\hline & $\operatorname{coxa}$ & $\begin{array}{l}\text { tro- } \\
\text { han- } \\
\text { ter }\end{array}$ & & $\begin{array}{r}\text { atel- } \\
\text { la }\end{array}$ & & $\begin{array}{l}\text { méta- } \\
\text { tarse }\end{array}$ & tarse & total & \\
\hline I & 0,9 & 0,3 & 3,7 & 1,3 & 3,6 & 4,0 & 0,7 & 14,5 & $(13,3)^{*}$ \\
\hline II & 0,9 & 0,3 & 3,7 & 1,2 & 3,4 & 3,9 & 0,7 & 14,4 & $(12,9)$ \\
\hline III & 0,8 & 0,3 & 2,8 & 1,0 & 2,5 & 3,0 & 0,7 & 11,1 & $(10,0)$ \\
\hline IV & 0,9 & 0,3 & 3,9 & 1,2 & 3,2 & 3,8 & 0,7 & 13,8 & $(12,6)$ \\
\hline pattes mâchoires & 0,5 & 0,2 & 1,7 & 0,6 & 1,2 & - & 1,5 & 5,7 & $(5,0)$ \\
\hline
\end{tabular}

Position du Tm I inconnue, Tm IV 0,9.

La vulva se distingue par la grande extension apicale du spermathèque et par la présence d'une apophyse dorsale sur le valve inférieur. L'abdomen est ovale-allongé, blanchâtre. La région épigastrique est couverte d'un scutum presque circulaire, englobant les quatre stigmates et l'orifice génital. Il contourne la base de l'abdomen et atteint environ le tiers de la longueur

* Les valeurs entre parenthèses indiquent le total moins les coxae et trochanters pour faciliter la comparaison avec les données d'autres publications.

Planche 3. 13) Rhode scutiventris. Monts Cantabres, vulva, dorsale - 14) $R$. biscutata, Edough, id. - 15) R. magnifica, id. - 16) R. magnifica - 17) id. 13, 14, x 72,5; $15, \times 110 ; 16,17$, x 22 . 


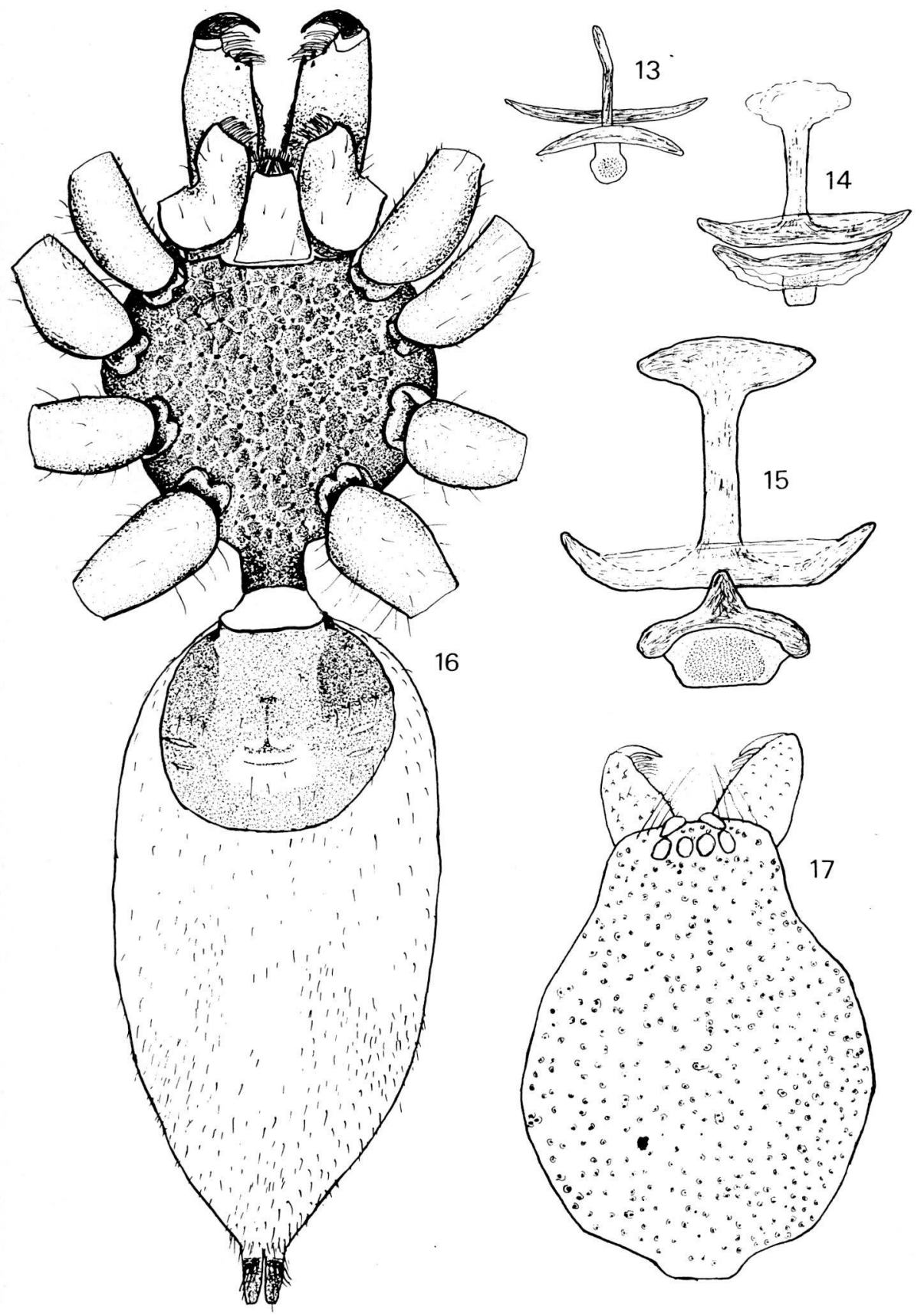


abdominale. Le côté dorsal de l'abdomen ne présente qu'une petite plaque triangulaire faiblement chitinisée. Les filières inférieures sont légèrement plus courtes et plus grosses que les supérieures, celles-ci sont munies d'un segment distal d'un tiers de la longueur du segment proximal.

L'espèce la plus proche semble être le $R$. biscutata, dont notre espèce en diffère par sa taille, sa partie céphalique plus étroite, ses chélicères plus minces et moins géniculées, la longueur de ses pattes, la forme de sa vulva et son scutum ventral plus court.

Rhode magnifica a été trouvée dans la partie antérieure éclairée d'une grotte glacière à courte distance de la glace. Elle était cachée dans les fentes entre les fragments de la roche friable dolomitique. La grotte est située à une altitude de $1000 \mathrm{~m}$ environ.

RHODE STALITOIDES spec. nova (pl 1: 3.5; pl 2: 9; pl 4; pl 6: 25)

Grotte "Megara", Lanište, montagne Bjelašnica. Bosnie. 1 ô leg. P. R. Deeleman, 1 iô subad. leg. C. L. Deeleman, 7-VIII-1973

Description. - Longueur du céphalothorax + abdomen 4,7 $\mathrm{mm}$, céphalothorax $2,1 \mathrm{~mm}$ long, 1,6 $\mathrm{mm}$ large, largeur de la partie céphalique $1,2 \mathrm{~mm}$, longueur du pédicule $0,2 \mathrm{~mm}$. Le céphalothorax, le sternum et les appendices sont rouge-jaunâtres comme chez Stalita. La tête est large, le céphalothorax étant peu atténué en avant (fig. 18). Les yeux sont totalement absents, la strie thoracique médiane est très peu développée, le lobe postérieur est faible. Le céphalothorax et la face antérieure des chélicères sont lisses, dans la région oculaire se trouvent quelques crins longs et fins dirigés vers l'avant. Les chélicères sont presque deux fois plus longues que larges au milieu, la géniculation posterieure est moins forte que chez les espèces occidentales et les autres espèces aveugles. Les marges antérieures et postérieures sont munies de deux dents égales, les marges étant bien séparées; l'échancrure est courte, presque transversale. Les lames-maxillaires sont très légèrement convergeantes distalement et s'inclinent faiblement sur la pièce labiale. Le sternum est lisse, le prolongement tubiforme n'est pas fermé dorsalement (fig. 3), son bord ventral est échancré en forme de V. Les pattes ambulatoires sont longues et grèles, les mensurations en $\mathrm{mm}$ sont les suivantes:

$$
\begin{aligned}
& \text { coxa tro- fémur patella tibia méta- tarse total } \\
& \text { chanter }
\end{aligned}
$$

$\begin{array}{lllllllll}\text { I } & 0,9 & 0,2 & 2,4 & 1,1 & 2,2 & 2,1 & 0,6 & 9,5 \\ \text { II } & 0,8 & 0,2 & 2,4 & 1,1 & 2,2 & 2,1 & 0,6 & 9,4 \\ \text { III } & 0,6 & 0,2 & 2,0 & 1,0 & 1,8 & 2,0 & 0,6 & 8,2 \\ \text { IV } & 0,8 & 0,2 & 2,6 & 1,1 & 2,2 & 2,4 & 0,6 & 9,9\end{array}$

Le Tm I est 0,71 ; Tm IV? 

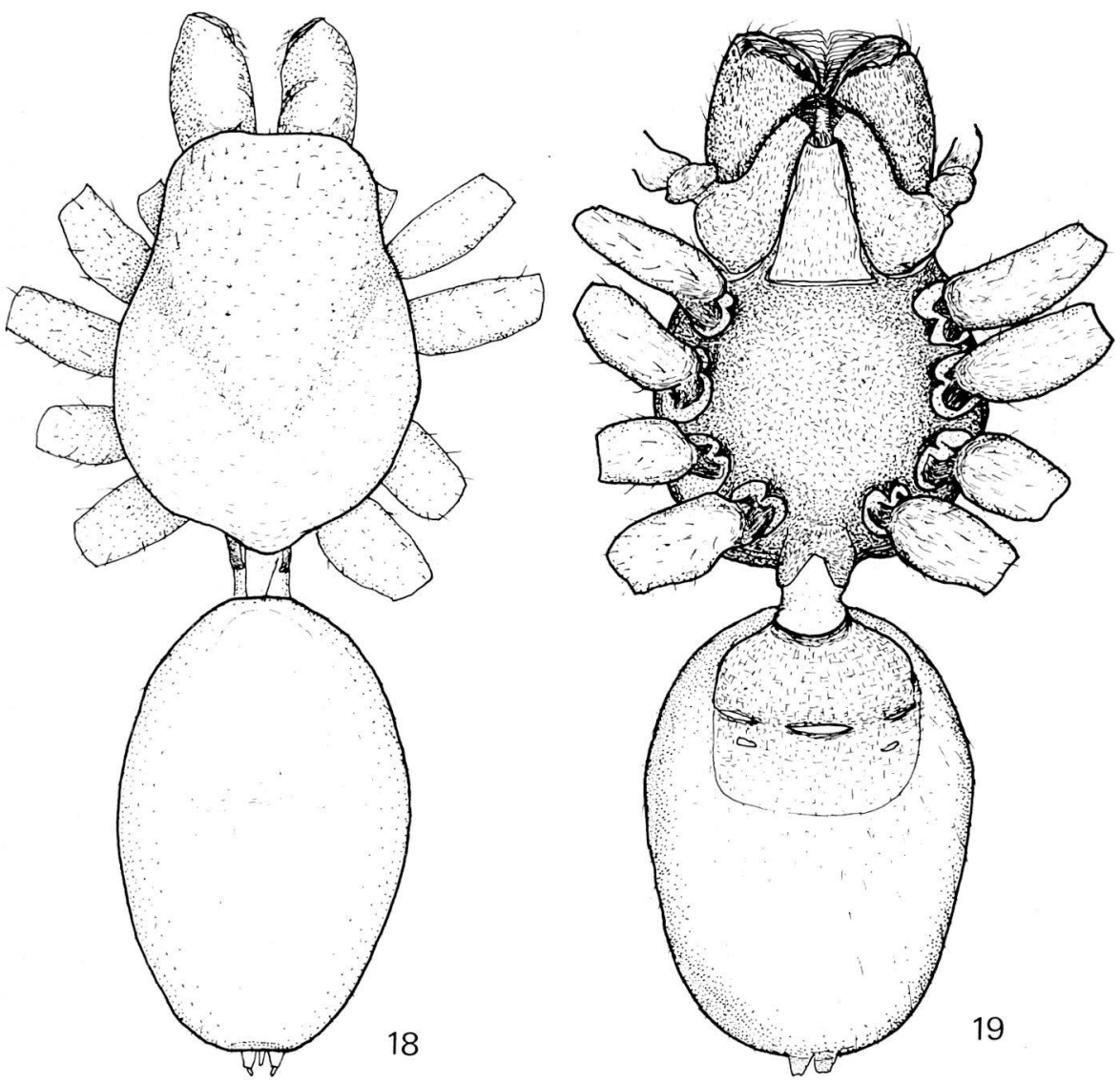

Panche 4. 18, 19) R. stalitoides, x 22.

Les griffes inférieures sont plus longues que la moitié des griffes supérieures (adaptation au milieu?). Le scutum dorsal comprend la moitié de la longueur abdominale et la moitié de sa largeur, le scutum ventral s'étend sur la moitié de la longueur de l'abdomen et sur toute sa largeur et il contourne la base de l'abdomen. Ils sont plus minces et clairs que chez les autres espèces, chez l'individu immature ils sont absents. Les filières sont comme chez l'espèce précédente.

Patte-mâchoire: fémur $1,1 \mathrm{~mm}$, patella $0,6 \mathrm{~mm}$, tibia $0,7 \mathrm{~mm}$, tarse $0,6 \mathrm{~mm}$, bulbe $0,6 \mathrm{~mm}$. (En comparaison, chez $R$. biscutata ot ces mensurations sont respectivement 1,2 - 0,5-0,7-0,9-0,7 et chez $R$. scutiventris 0,6 - 0,3 - 0,3-0,5$0,4)$. On observe une demi-douzaine de processus spiniformes transparents sur un champ subapical non chitinisé du bulbe (chez biscutata il y en a 2-3, chez 
scutiventris 5-6, chez tenuipes aucun, chez Stalita taenaria une dizaine).

Rhode stalitoides a été capturée dans la salle terminale entièrement concrétionnée de la grotte (température de $5,5 \mathrm{C}^{\circ}$ au mois d'août), errant sur les stalagmites. Elle se distingue de $R$. subterranea par le moindre développement du lobe thoracique postérieur, par les pattes beaucoup plus longues et les griffes tarsales impaires plus longues, puis par le scutum dorsal plus court et plus étroit. Elle se distingue de $R$. aspinifera par le plus petit lobe thoracique postérieur, par la disposition des dents des chélicères, la forme des lamesmaxillaires, les pattes beaucoup plus longues et les scuta plus petits. Le palpe mâle rappelle beaucoup celui de scutiventris mais le tarse est relativement plus court. $R$. stalitoides s'approche du genre Stalita par la tête large tronquée, le faible développement du lobe thoracique, le pédicule relativement court, la réduction des scuta et par la patte-mâchoire du mâle dont le tarse est plus court que le tibia.

RHODE ASPINIFERA (Nikolić) (pl 5; pl 6: 27) Typhlorhode aspinifera Nikolić, 1963

Jama Osojica, Belsko près de Postojna, 1 q , leg. 14-IX-1952. Hrenovi tali près de Postojna, au dessous des pierres profondément enfoncées, $3 q$, leg. E. Pretner 5-V-1954.

Lenčkova jama, Bukovje, près de Postojna, $1 \stackrel{q}{q}$, leg. E. Pretner 11-II-1958. Jama v bukovih dolinah, Trnovo (Slovénie occidentale), 1 \& , leg. E. Pretner 30-VI-1974.

Nikolić a décrit cette espèce sans en donner de figures. Par erreur, il signale l'absence du lobe postérieur au thorax. Pour éviter une confusion, je présente ci-dessous une redescription.

Description: (Hrenovi tali). - Longueur du céphalothorax + abdomen 4,7 mm, céphalothorax 1,9 $\mathrm{mm}$ long, 1,4 mm large, largeur de la partie céphalique 0,7 $\mathrm{mm}$, longueur du pédicule $0,2 \mathrm{~mm}$. La couleur des parties chitinisées est d'un orange vif, les yeux et les stries thoraciques font complètement défaut. Le lobe postérieur du thorax est bien développé. Le céphalothorax, sternum, chélicères et les parties proximales des pattes ambulatoires sont couverts de petits mamelons piligères. L'anneau sternal enveloppant le pédicule n'est pas fermé dorsalement (fig. 22). Les chélicères sont deux fois plus longues que larges au milieu, fortement géniculées postérieurement. L'échancrure est diagonale, trois fortes dents coniques sont rangées presqu'en ligne droite, les deux basales conniventes, la plus distale séparée d'un diamètre environ. Une quatrième dent minuscule se trouve devant les deux basales. Les lames-maxillaires sont droites, leurs bords extérieurs à peine convexes, tronqués droit distalement; ils ne s'inclinent pas sur la pièce labiale.

Les mensurations en $\mathrm{mm}$ des pattes sont les suivantes: 

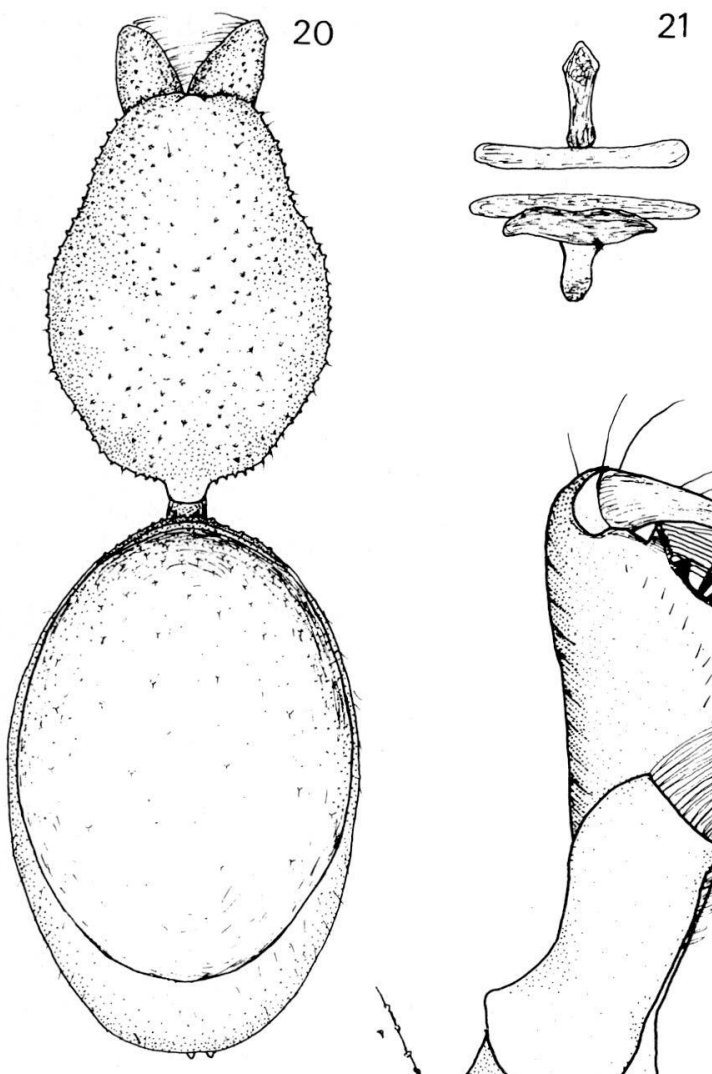

22

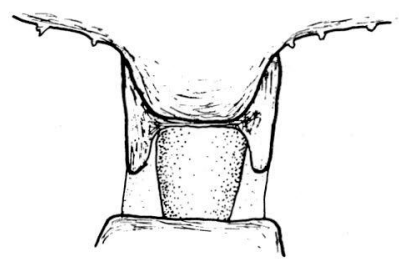

Planche 5. 20) R. aspinifera (Nikolić), Hrenovi Tali - 21) id., vulva, dorsale - 22) id., lobe postérieur du céphalothorax + pédicule -23 ) id., pièces buccales et partie antérieure du sternum. 20, x 22; 21, x $110 ; 22-23$, x 72,5 .

\begin{tabular}{|c|c|c|c|c|c|c|c|c|}
\hline & coxa & $\begin{array}{l}\text { tro- } \\
\text { han- } \\
\text { ter }\end{array}$ & & $\begin{array}{r}\text { tel- } \\
\text { la }\end{array}$ & & & arse & tota \\
\hline & 0,6 & 0,1 & 1,6 & 0,9 & 1,3 & 1,2 & 0,4 & 6,1 \\
\hline & 0,6 & 0,1 & 1,5 & 0,8 & 1,2 & 1,1 & 0,4 & 5,7 \\
\hline & 0,4 & 0,1 & 1,2 & 0,6 & 0,9 & 1,1 & 0,4 & 4,7 \\
\hline & 0,5 & 0,1 & 1,5 & 0,7 & 1,3 & 1,4 & 0,4 & 5,9 \\
\hline mâchoire & 0,3 & 0,1 & 0,6 & 0,4 & 0,4 & - & 0,4 & 2,2 \\
\hline
\end{tabular}


La plupart des Tm I manquent, chez un exemplaire le Tm I est 0,78; Tm IV est 0,68 .

Les griffes inférieures des tarses (fig. 27) sont petites, beaucoup plus courtes que la moitié des griffes supérieures. Les scuta dorsal et ventral s'étendent sur presque la totalité de l'abdomen, ils portent quelques fines soies.

Cette espèce a été capturée dans les environs de Postojna, dans les grottes et aussi à l'extérieur sous des pierres profondément enfoncées, dans le fond des

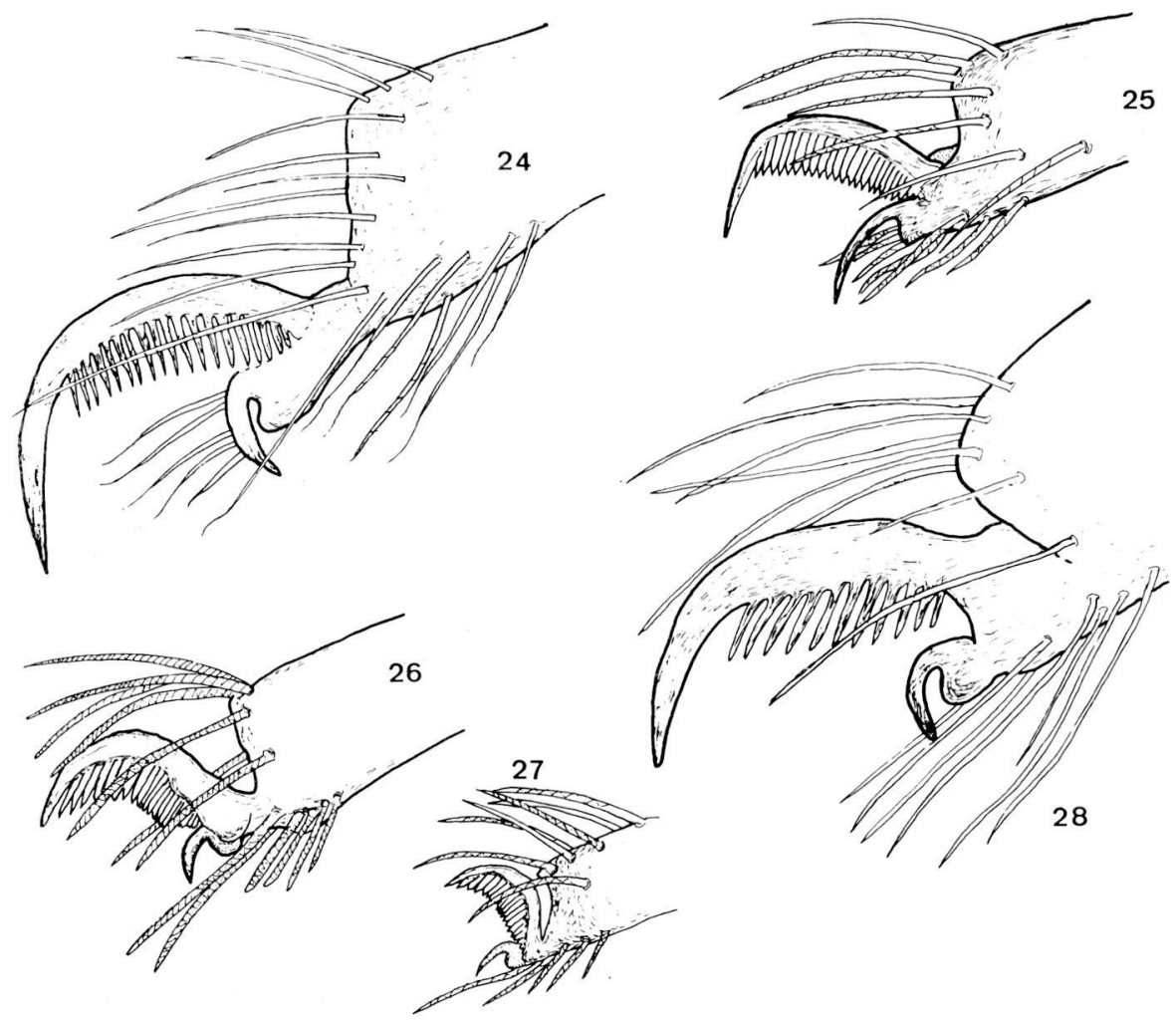

Planche 6. 24) Stalita taenaria Schiödte, ô. Križna jama, extrémité du tarse I - 25) $R$. stalitoides, id. -26) $R$. magnifica, id. - 27) $R$. aspinifera, Hrenovi Tali, id. - 28) Stalagtia hercegovinensis (Nosek), $q$, Debeljin pećina, id. 24-28, x 110.

forêts. Un exemplaire fût trouvé dans une grotte dans le "Tarnowaner Wald" dans le Sud-Ouest de la Slovénie, où il se tenait sur une roche faiblement éclairée près de l'entrée. Géographiquement l'espèce paraît être largement séparée de $R$. subterranea de l'Herzégovine à laquelle elle ressemble beaucoup. Elle se distingue de cette dernière par la dent basale antérieure des chélicères qui est beaucoup plus petite que les autres dents, par les lames- 


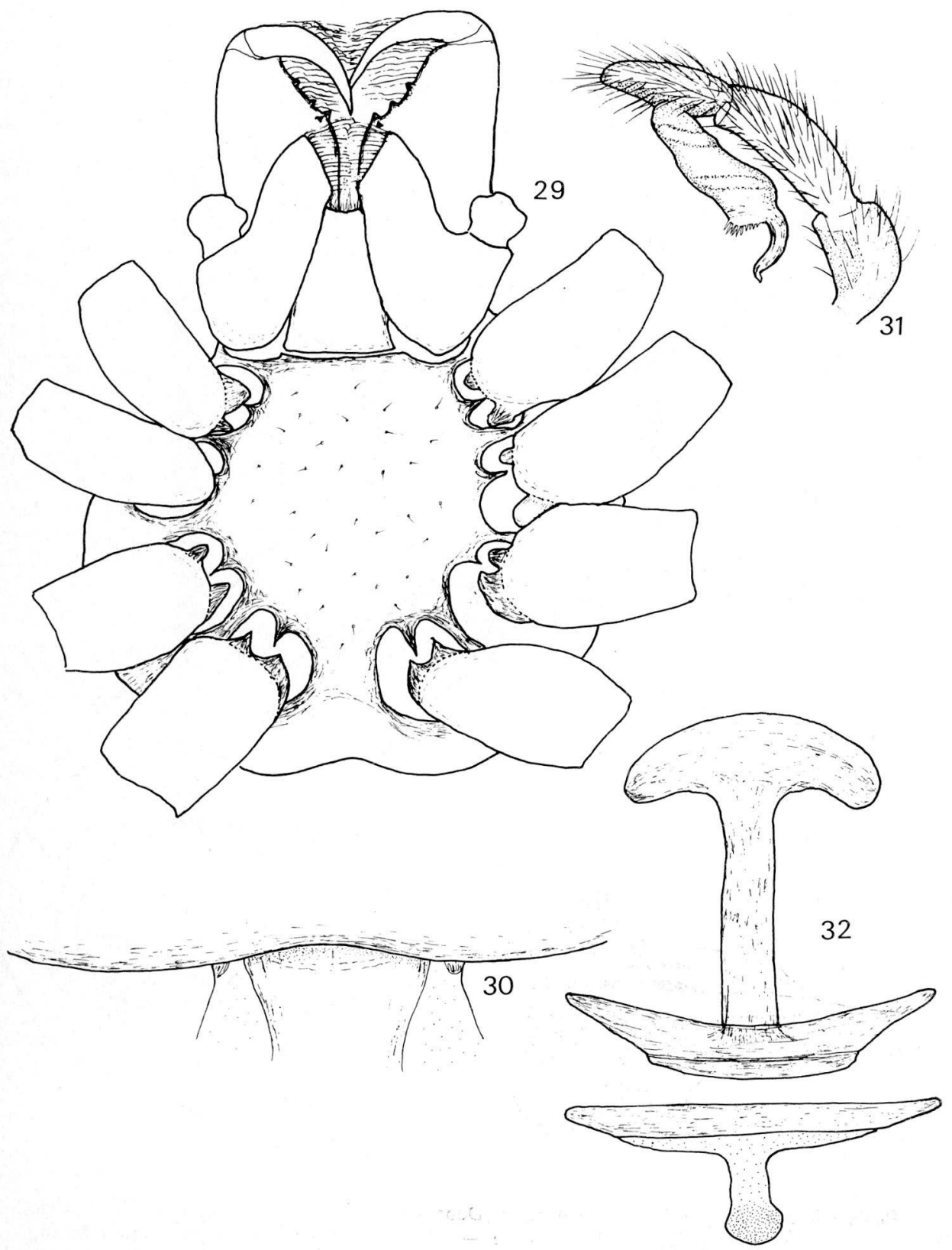

Planche 7. 29) Stalita taenaria ô (Križna jama), sternum et appendices - 30) ịd., bord postérieur du céphalothorax - 31) id., patte-mâchoire - 32) id.,, , Mrzla jama, vulva, dorsale. 29,31, x $22 ; 30$, x 72,5; 32, x 110 . 


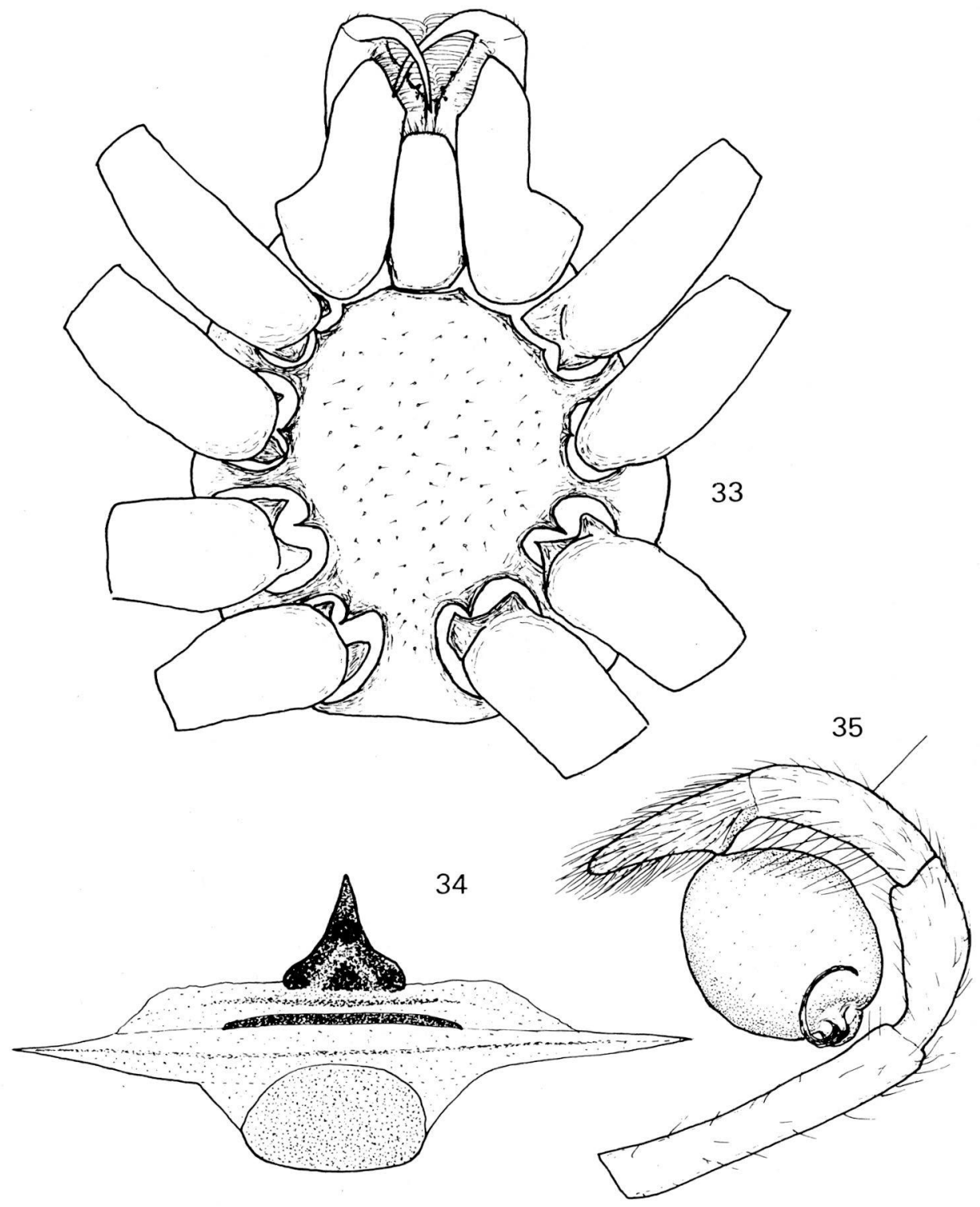

Planche 8. 33) Stalagtia hercegovinensis, ồ (Debeljin pećina), sternum et appendices - 34) id., q (Doli pećina), vulva, dorsale - 35) id., $\hat{\delta}$, (Debeljin pećina), patte-mâchoire. 33,35, x 22; 34, x 110. 
maxillaires plus larges distalement, par la première paire des pattes ambulatoires plus longue que la quatrième paire, par l'absence d'une branche transversale au spermathèque et par les scuta dorsal et ventral plus étendus.

\section{DISCUSSION}

Bien que notre connaissance de Rhode s'appuie sur très peu d'individus et que le nombre d'espèces connues n'est sans doute qu'une fraction du nombre existant, les informations retenues par la découverte d'espèces nouvelles yougoslaves permettent mieux qu'auparavant de préciser la position systématique au sein des Dysderidae. Ces Araignées archaïques présentent quelques caractères uniques qui les éloignent de tous les autres genres. Il faut insister sur le fait qu'on connait actuellement l'existence de quatre espèces endémiques yougoslaves, dont trois anophtalmes, contre trois espèces ailleurs, toutes oculées, et j'incline à penser que les Rhode soient autochtones dans ces contrées.

Leur parenté avec les espèces du genre Stalita et genres affines, Parastalita, Mesostalita et Stalitella, tous cantonnés dans la partie septentrionale de la Yougoslavie (Deeleman-Reinhold, 1971) se traduit par le fait qu'ils ont en commun un nombre de caractères constituant d'excellents critères phylogénétiques. En premier lieu ce sont les organes copulateurs mâles et femelles, auxquels s'ajoutent le sternum large et convexe à bord antérieur divisé en trois portions dont celle au milieu la plus large (chez Harpactea/Stalagtia il y a trois portions égales, fig. 33) les liaisons chitineuses entre les coxae I et celles des pattesmâchoires (Kratochvil, 1970) et la possession de lanières enroulées hélicoïdalement (?), surtout aux extrémités des tarses. Ces observations permettent de porter quelques modifications dans la classification proposée par Cooke (1965). Cet auteur divise les Dysderidae en quatre tribus: Dysderini (Tedia, Stalitochara, Dysdera, Harpactocrates), Harpactini (Dasumia, Harpactea, Holissus, Stalita), Orsolobini (Orsolobus) et Rhodini (Rhode, Harpassa et Typhlorhode). La connaissance acquise au cours d'une étude comparative de nombreuses nouvelles formes de Dysderides yougoslaves hypogées et épigées (Deeleman-Reinhold, 1977) me conduit à supprimer le groupe Rhodini et à le remplacer par le groupe des Stalitini, composé des Stalita et genres apparentés yougoslaves et les Rhode s.l. Les genres Stalagtia et Folkia, jusqu'à 1970 inclus dans le genre Stalita, sa rattachent facilement aux Harpactini.

Il y a des raisons de croire que les Stalitini, comme tant d'autres groupes de cavernicoles surtout terrestres, aient pris naissance dans le Péninsule Balkanique et nous ne devons pas voir en eux des relictes d'une faune très ancienne à large dispersion. Les Stalita se seraient installés dans les grottes à une période reculée, tandis qu'un rameau de Rhode aurait franchi la Méditerranée pour coloniser l'Afrique du Nord.

Nous pensons maintenant que la Yougoslavie soit une région privilégiée ou les contacts avec le milieu souterrain, en collaboration avec les vicissitudes du climat pendant de longues périodes géologiques, ont favorisé de nombreuses 
installations locales en milieu souterrain. Ceci s'est traduit par une richesse de formes adaptées à ce milieu dans de nombreux groupes.

Je veux exprimer ici ma gratitude sincère à l'hospitalité du Muséum National d'Histoire Naturelle à Paris et à l'obligeance de M. Michel Hubert, qui m'ont rendu possible d'utiliser les types des espèces occidentales de Rhode pour cette étude comparative.

\section{RÉSUMÉ}

Description de Rhode magnifica n. sp. d'une grotte du Monténégro et de $R$. stalitoides d'une grotte Dosnienne. Redescription de $R$. aspinifera (Nikolić). L'auteur réunit avec le genre Rhode les genres Harpassa et Typhlorhode en un seul genre Rhode et en donne une diagnose. Ce genre présente des liens de parenté avec le genre Stalita du Nord de la Yougoslavie. On peut supposer que ces deux groupes ont pris naissance dans la Péninsule Balkanique en dérivant d'un ancêtre commun.

\section{BIBLIOGRAPHIE}

BRIGNOLI, P. M. 1975. Ragni d'Italia 23. Nuovi dati su alcune Haplogyne. Boll. Soc. Ent. Ital. 107 (9-10): 170-178.

COOKE, J. 1965. Spider genus Dysdera (Araneae, Dysderidae). Nature 205: 1027-1029.

DEELEMAN-REINHOLD, C. L. 1971. Beitrag zur Kenntnis der höhlenbewohnenden Dysderidae (Araneida) aus Jugoslawien. Razprave Slov. Ak. Znan. Umetn. 14(4): 93-120.

- 1977. On the affinities of some Yugoslavian troglobitic spiders. Proc. 7th Int. Spel. Congr. Sheffield : 146-150.

KRATOCHVIL, J. 1935. Ein neuer Vertreter der Gruppe Rhodeae aus den Balkanischen Höhlen. Studien aus dem Geb. der allg. Karstforsch., wiss. Höhlenk. und Nachbargeb. Biol. Ser. 1: 1-10.

1970. Cavernicole Dysderae. Acta Sc. Nat. Brno 4(4):1-62.

LEHTINEN, P. T. 1975. Notes on the clássification of Araneae. Proc. 6th Int. Arachn. Congress Amsterdam: 26-29.

NIKOLIC, F. 1963. Pauci iz nekih pećina Slovenije. (Die Spinnen aus einigen Höhlen Sloweniens). 3ième Congr. Youg. de Spéléologie, Sarajewo: 157-167.

1966. Beitrag zur Kenntnis der Spinnenwelt in der Umgebung von Dubrovnik. Folia ent. Hung. 19(24): 441-453.

SIMON, E. 1882. Description d'espèces et de genres nouveaux de la famille des Dysderidae. Ann.

Soc. Ent. France: $201-240$.
1893. Description d'espèces et de genres nouveaux de l'ordre des Araneae. Ann. Soc. Ent. France: 62: 299-330.

1893a. Histoire naturelle des araignées. Tome I: 308-322. 